\title{
ContentSelect
}

Annals of Botany Volume 104 Number 52009

\section{John Bryant takes a closer look at some of this month's Original Articles}

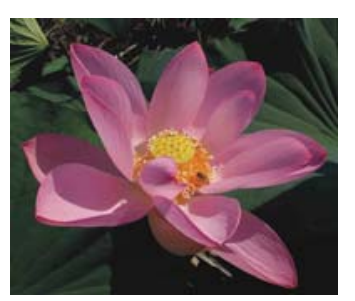

\section{Beetles drive Lotus but bees buzz in}

Outside of my 'professional' interests in biochemistry and molecular biology I am especially fascinated by floral biology, including the development during evolution of floral traits in relation to pollinators. It is widely held that stabilizing selection by pollinators has led to the development of 'pollination syndromes'. Thus, examination of floral morphology and physiology may give clear clues as to the identity of the pollinators of a particular species. It is this idea that has informed the work of Li and Huang at Wuhan (pp. 845-851). The Asian sacred lotus (Nelumbo nucifera) is a 'living fossil', a basal angiosperm placed right at the bottom of the angiosperm phylogenetic tree. It has homoeothermic flowers that appear to be strongly adapted for pollination by beetles (cantharophily); however, the question is whether specimens living today are actually beetle-pollinated. The authors identified visitors to flowers in 11 wild populations in locations across China and in a cultivated population at Wuhan. The results of this survey were very clear. Only at Wuhan and Honghu were beetles observed as floral visitors, despite the presence of relevant species of beetle in the other regions. It would be interesting to know why the beetles ignored the Nelumbo flowers in these regions. By contrast, in all populations, including Wuhan and Honghu, bees, flies and thrips were seen to be floral visitors. Observation of pollen deposition combined with analyses of seed-set in flowers enclosed in mesh bags with different mesh sizes indicated that, despite frequency of visits, thrips were not effective pollinators. In most populations therefore, bees and flies are important pollinators. In Wuhan and Honghu, beetles were certainly effective pollinators but again bees played a significant role. Nevertheless, floral features still point strongly to beetles as the 'ancestral' pollinators. However, flower structure does not exclude other insects, enabling the Asian sacred lotus to exploit a range of contemporary pollinators.

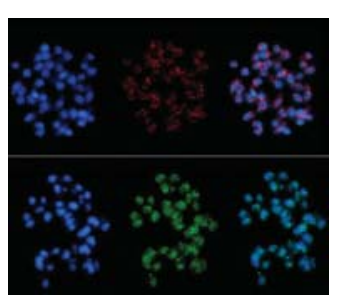

\section{Retro repeats demonstrate DNA diversity}

It is well known that within the Angiosperms genome size varies hugely. Most of the variation involves non-coding repetitive DNA sequences. Analysis of these sequences has been used to study both genome evolution and relationships between species and genera (e.g. Sibson et al. 1991. Sequence organization of simple, highly repetitive DNA elements in Brassica species. Journal of Experimental Botany 42: 243-249), and has now been applied by Begum et al. (Dhaka, Bangladesh and Dresden, Germany; pp. 863-872) to the orchid genus Dendrobium. This is a very large genus (nearly 1200 species) with very broad interspecific morphological diversity and a wide geographical range. Taxonomic classification is currently based on morphological characters and chloroplast DNA sequences but, as the authors state, classification of many Dendrobium species is ambiguous. Sixteen species are known in Bangladesh, including commercially valuable ornamentals and some endangered species. Greater understanding of interspecific relationships is therefore important. The authors made a plasmid library of rapidly renaturing DNA from D. moschatum and then used the library in Southern hybridizations with genomic DNA in order to pull out highly repetitive sequences. Six of the eight clones thus obtained were Arabidopsis-like telomeric repeats; one was an array of the dinucleotide GA (termed DmoO11) while the last was a novel, highly amplified, dispersed 420bp repeat termed DmoF14. This sequence was not previously present in the databases and may represent the long terminal repeat (LTR) of an ancient orchid retro-element. It is highly conserved in the genus Dendrobium while the equivalent elements in Nagaliella and Phalaenopsis show some sequence divergence from Dendrobium. FISH analysis revealed, as expected, that the telomere sequence is located at chromosome ends. However, in $D$. aphyllum, two chromosome pairs also exhibit interstitial locations for this sequence, implying a degree of chromosome re-arrangement. Both the dinucleotide repeat and the orchid-specific 420-bp sequence are present throughout the genome but there is variation between species with respect to specific chromosomal location. This again implies that some sequence re-arrangements have occurred during evolution of the genus. 


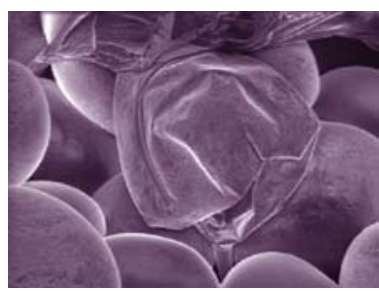

\section{ABA addition affects Atriplex actions}

There is no doubt that we need to grow more food, using less water and on less land, and that more of the land under agriculture will be salt-contaminated. Thus we agree with Ben Hassine et al. (Tunis and Louvain-la-Neuve, Belgium; pp. 925-936) that understanding drought and salt tolerance is very important. These two environmental factors have some overlapping effects but also elicit specific responses. Studying a xero-halophyte such as Atriplex halimus is thus very useful. The authors exposed seedlings from an inland, waterstress-tolerant population and a coastal salt-resistant population to 40 or $160 \mathrm{~mm} \mathrm{NaCl}$ or to $15 \%$ PEG (water-stress treatment). Growth of the coastal population was less affected by $\mathrm{NaCl}$ than growth of the inland population. The reverse was true for water stress. These responses were paralleled by ABA accumulation: the coastal population accumulated more $\mathrm{ABA}$ than in the inland population in response to $\mathrm{NaCl}$ and the reverse was seen in response to water stress. Both $\mathrm{NaCl}$ and $\mathrm{PEG}$ elicited the accumulation of the osmo-protectants proline and glycine-betaine in both populations, with greater concentrations of proline in the inland population and greater concentrations of glycine-betaine in the coastal population. Application of exogenous ABA $(50 \mu \mathrm{M})$ improved water-stress-resistance in the coastal population via its action on regulation of stomatal aperture; it improved NaCl-resistance in inland populations by promoting excretion of $\mathrm{NaCl}$ through the salt bladders. ABA also stimulated proline accumulation but only in the response to water-stress. Further, polyamines, especially spermidine and spermine, were involved in the response to $\mathrm{NaCl}$. Both accumulated in response to salt treatment and this response was increased in the presence of ABA. Inhibition of polyamine synthesis prevented accumulation of spermine and spermidine and also interfered with salt excretion. The authors speculate that polyamines interact with ATPase and/or with ion channels. Overall, then ABA is involved in both salt and water-stress responses acting via common and stress-specific pathways.

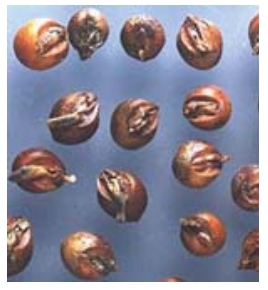

\section{Grain germination geared to ABs expression}

In general, crop plants have been selected to exhibit low levels of dormancy, thus expediting use of seeds soon after harvest; however, this may have disadvantages. In cereals for example, crop losses may occur through pre-harvest sprouting (PHS). In an investigation of this problem, Rodriguez et al., at Buenos Aires (pp. 975-985) have worked with two lines of Sorghum bicolor differing markedly in their susceptibility to PHS; we focus here on a selection of their results. The difference between the two lines was seen clearly in germination tests on caryopses conducted 30 and 47 days after pollination (DAP). Surprisingly perhaps, the marked differences in germinability were not mirrored by differences in ABA content. Rather, it was differences in the signalling pathway downstream of ABA that were correlated with susceptibility to sprouting, raising the possibility of differences in sensitivity to ABA. In the sprouting-resistant cultivar, expression - measured by mRNA abundance - of five genes involved in ABA signalling (three transcription factors and two protein kinases) increased markedly when 30-DAP caryopses were incubated in water. This happened to a much lesser extent (or not at all) in the sprouting-susceptible cultivar. For the transcription factor SbAB15, this difference was confirmed at the level of protein abundance. These differences between the two lines disappeared after physiological maturity when, in both cultivars, incubation of caryopses led to a rapid decline of all transcripts and of $\mathrm{SbAB} 15$ protein. The situation is made more complex by the role of the seed coat: isolated 30-DAP embryos from the sprouting-resistant line showed approx. $30 \%$ germination after $12 \mathrm{~h}$ incubation in water. This was inhibited by ABA, but ABA-treated embryos were no different from controls in respect of SbAB15 transcript or protein (although differences in protein phosphorylation cannot be ruled out). For this transcription factor at least, in grains developing in situ, both ABA and the seed coat may be involved in its regulation.

Professor J. A. Bryant University of Exeter, UK E-mail j.a.bryant@exeter.ac.uk 